\title{
Percepción de la Seguridad Alimentaria de los hogares del municipio San Ramón, Matagalpa 2014-2015
}

\author{
MSc. Carmen María Flores Machado \\ Docente Investigadora \\ UNAN- MANAGUA, FAREM- MATAGALPA \\ carmenflores280165@yahoo.com
}

Palabras clave: Territorio, Seguridad Alimentaria, Percepción, Medios de Vida

\section{RESUMEN}

Ste estudio tiene la importancia de conocer los activos que las comunidades de San Ramón

E tienen a su disposición para satisfacer sus necesidades básicas de alimentación, desde la percepción de la seguridad alimentaria, que le permitan alcanzar su bienestar nutricional. El municipio de San Ramón es caracterizado con una incidencia de pobreza y vulnerabilidad nutricional. La investigación tiene una perspectiva holística en la cual se fundamenta la propuesta de la sustentabilidad ligada a la acción del hombre en relación a su entorno. El presente estudio es descriptivo y observacional, con un enfoque mixto, es decir cualitativo-cuantitativo. La muestra estuvo constituida por 301 hogares, en las comunidades de Monte Cristo, Los Limones, Mil Bosque, El Horno I, Yucul Central, San Ramón, El Plomo, Mata Palo, La Garita, Siares el Toro, Siares La Virgen, El Naranjo, Trentino Obrero, San Pablo, Sabana Grande, Wabule, El Jícaro II, Azancor I, El Esquirín y del casco urbano, los barrios La Cruz y Álvaro Castillo. La prevalencia de inseguridad alimentaria en hogares visitados en el área urbano y rural de San Ramón, es del 89 \%, y el 19 \% tienen seguridad alimentaria. El 69\% tienen jefe de familia varón y 31 \% mujer, integrado por familia extendida con sobrinos, abuelos y cuñados. En general, existe inseguridad alimentaria en los hogares donde hay niños y jóvenes, en los que se observa la preocupación por los alimentos sino su afectación en la calidad y cantidad de alimentos y sino también en el padecimiento de hambre.

\section{INTRODUCCION}

El siglo XXI desafía al medio rural con nuevos y complejos retos, hace frente al cambiante papel de la ruralidad en el desarrollo, logra el equilibrio entre la seguridad alimentaria y las preocupaciones ambientales, y encuentra respuestas a la seguridad energética, son algunos de los retos. La producción de alimentos en los últimos años ha sido afectada por el cambio climático, la menor disponibilidad de tierras para la agricultura, declinación del crecimiento de los rendimientos por hectáreas y competencia creciente por el recurso agua y combustible fósil, son problemas urgentes que atender por los gobiernos y la humanidad (IICA, 2009). 
En los últimos años, la seguridad alimentaria se ha posicionado como una prioridad en la agenda política tanto anivel global, como en América Latina y el Caribe. Una agenda de políticas públicas para enfrentar los principales desafíos de la región debe incluir: un rol político más activo de la región en los mecanismos de gobernanza de la seguridad alimentaria y nutricional a escala mundial; políticas de largo plazo para abordar estructuralmente la transformación de los patrones de producción y consumo, aumentando la inversión en agricultura y poniendo prioridad en la inclusión de la agricultura familiar; medidas para adaptar la agricultura al cambio climático, y lograr una mayor transparencia y competencia en los mercados agroalimentarios (FAO, 2011).

En el 2011, América Latina y el Caribe afronta un nuevo nivel de los precios de los alimentos, superior al de los últimos treinta años y alrededor de un $40 \%$ mayor que cuatro años atrás. A estas alzas se le suma una mayor volatilidad. Cabe recordar, que los cereales son la principal fuente de calorías para los habitantes de la región y del mundo, y dos de los más importantes -el trigo y el maíz- han aumentado sus precios en $62 \%$ y $104 \%$ respectivamente solo en el último año (FAO, 2011).

Según el Instituto de Nutrición de Centroamérica y Panamá y la Organización Panamericana de la Salud (2013), los problemas de Inseguridad alimentaria y nutricional tienen profundas raíces combinadas en diversas formas de acuerdo al propio contexto, pero sus causas fundamentales están basadas en un inadecuado suministro de alimentos, bajo poder adquisitivo familiar, deficientes condiciones sanitarias y escasos conocimientos en salud, alimentación y nutrición.

En ese sentido, la desnutrición, producto prolongado de un inadecuado suministro de alimentos en cantidad y calidad, provoca un subdesarrollo humano que, en el caso de Centroamérica, afecta principalmente a niños y niñas menores de cinco años y a mujeres de edad fértil. En el caso de la niñez menor de cinco años, el retardo en talla para la edad, indicador de desnutrición crónica, es el problema nutricional de mayor prevalencia en Centroamérica (PRESANCA, 2010).

En los departamentos del corredor seco, la desnutrición crónica en menores de cinco años (ENDESA 2011/12) refleja la magnitud del problema, superando la media nacional hasta en 6.5 puntos porcentuales en la severa (<3DE) y en 12.2 puntos la total. Siendo los Departamentos de Jinotega, Madriz, Nueva Segovia, Matagalpa y Managua los más afectados de 29.5\% a 14.2\%.

El proceso de desarrollo territorial con enfoque de la Seguridad Alimentaria y Nutricional incluye proyectos con visión integral vinculados a los capitales del Desarrollo Humano, Social, Productivo, Natural y Físico para mejorar las condiciones de Inseguridad Alimentaria y Nutricional del Municipio de San Ramón que integran los municipios de la zona seca, caracterizados por ser municipio de alta vulnerabilidad nutricional.

Actualmente, se reconoce que la disponibilidad de alimentos no es garantía del acceso a los mismos. Algunos estudios evidencian disponibilidad suficiente de alimentos relacionada con altos niveles de subnutrición y desnutrición infantil, como consecuencia de una alta desigualdad en el acceso a los alimentos, explicada por los bajos ingresos, que se traduce en un bajo poder de compra de alimentos en el mercado. Esta situación es particularmente notoria en Nicaragua.

La presente investigación analiza la percepción de la seguridad alimentaria y nutricional de los hogares de San Ramón y su relación con los medios de vida de los hogares. También, identifica los medios de vida que poseen y cómo enfrentan la situación alimentaria. 


\section{MATERIALES Y MÉTODOS}

El presente estudio es descriptivo y observacional, con un enfoque mixto, es decir cualitativocuantitativo.

El Municipio de San Ramón Matagalpa pertenece al departamento de Matagalpa.Tiene una extensión territorial de $424 \mathrm{Km}^{2}$, lo que representa el 7\% del territorio del departamento. Posee una altura de 640.93 metros sobre el nivel del mar y una posición geográfica de latitud $12^{\circ} 55^{\prime}$ y longitud 85 50'. La precipitación anual fluctúa entre los 1600 - 2000 mm y no tiene canícula. Está situado en la Cuenca 55, Río Grande de Matagalpa. Los suelos son mayormente francos arcillosos y arcillosos, con erosión moderada y mayormente con cobertura agropecuaria. El municipio de San Ramón, con una extensión territorial de 424 kilómetros cuadrados, está ubicado en la zona central de Nicaragua, tiene una incidencia de la pobreza de $60.3 \%$ y una prevalencia de retardo en talla de $48.38 \%$, con un nivel de analfabetismo del $36.8 \%$, índice de servicios insuficientes del 33.1\%, índice de vivienda inadecuada del 6.7\%, índice de baja educación del $28.8 \%$ e índice de dependencia económica del 53,4\%. Este municipio se encuentra a 12 kilómetros de la cabecera departamental y a 145 de la ciudad capital. Dividida en 11 comarcas y 51 comunidades. El casco urbano con 9 barrios. Cuenta con una población cercana a los 35400 habitantes, y una densidad reportada de 83.7 habitantes por $\mathrm{Km}^{2}$.

El universo de estudio fueron 5653 hogares, ubicados en el área urbana y rural del municipio de San Ramón. El área urbana está dividida en 9 barrios y el área rural formada por 11 comarcas con 51 comunidades.

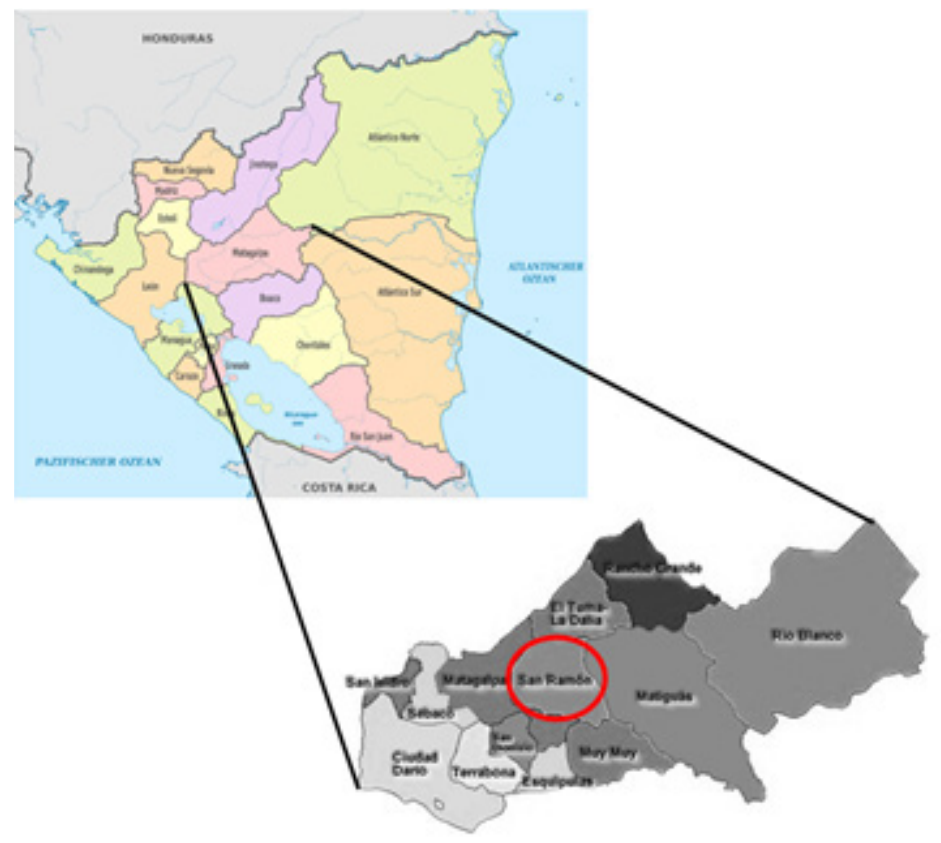

Gráfico 1. Zona de Estudio

Para determinar el tamaño de la muestra, se aplicó la fórmula de Mounch Galindo para poblaciones finitas y se obtuvo una muestra con un $95 \%$ de confianza y un $5 \%$ de error muestral a partir de la población de 5653 hogares, obteniendo un total de 301 hogares a encuestar. El procesamiento de la información se hizo a través del programa estadístico SPSSVersion25 para Windows. 
Para determinar la Inseguridad Alimentaria en el hogar, se aplicó la encuesta ELCSA (Escala Latinoamericana y Caribeña de Seguridad Alimentaria). Las preguntas que trae la escala se relaciona a la preocupación de que los alimentos se acaben en el hogar, la cantidad y calidad de alimentos disponibles en el hogar, y cuando no hay, qué estrategias utilizan para enfrentarla.

Consta de 15 preguntas, en la primera sección (P1 - P8) se cuestionan diversas situaciones que conllevan a la inseguridad alimentaria experimentadas en los hogares y los adultos de esos hogares; y una segunda sección (P9- P15) con preguntas referidas a condiciones que afectan a los menores de 18 años en el hogar. Cada pregunta está dirigida a indagar una situación diferente, por lo que se trata de preguntas excluyentes.

Utilizar esta escala va más allá de la percepción de los jefes de hogar, la que se refiere a situaciones objetivas autorreportadas que los integrantes del hogar experimentan, tales como, la reducción de la cantidad de alimentos servidos, la omisión de alguna de las comidas diarias, la presencia de hambre en alguno de los integrantes del hogar y la suspensión de comidas debido a la falta de dinero u otros recursos.

La escala ELCSA es un instrumento de medición indirecta de la seguridad alimentaria en el hogar, de bajo costo, que ha demostrado validez y confiabilidad en diversos canales de aplicación. En el nivel local hasta el nivel nacional, se ha tenido mucho éxito en comprensión de la distribución, causas y consecuencia de la inseguridad alimentaria en la región. Mide los grados de severidad de la inseguridad alimentaria (leve, moderada, severa), es un instrumento confiable que permite optimizar y focalizar alertas tempranas en políticas de prevención, según la FAO (mayo 2012).

La frecuencia de consumo de alimentos es un método para evaluar el patrón de consumo de alimentos a nivel familiar mediante un listado de 67 alimentos con frecuencia semanal.

Consentimiento Informado: Los jefes de los hogares participantes firmaron el consentimiento informado, el cual incluyó los principios éticos para las investigaciones médicas en seres humanos de la declaración de Helsinki.

\section{RESULTADOS Y DISCUSIÓN: TABLAS Y GRÁFICOS}

La prevalencia de inseguridad alimentaria en hogares visitados en el área urbano y rural de San Ramón es del $89 \%$, y el 19\% tienen seguridad alimentaria. La categoría de inseguridad alimentaria de moderado y severo representó el 52\% de los hogares y la inseguridad leve, con el 37\%, observándose una tendencia de aumentar los grados más severos que pueden afectar el estado nutricional de las personas.

Algunas características relevantes de estos hogares son, el 69\% tienen jefe de familia varón y $31 \%$ mujer, integrado por familia extendida con sobrinos, abuelos y cuñados. El $52 \%$ son mujeres y $48 \%$ varones, con un nivel de escolaridad de primaria incompleta para el $37 \%$ y secundaria incompleta con el 15\%; encontrándose un $17 \%$ de analfabetas. 


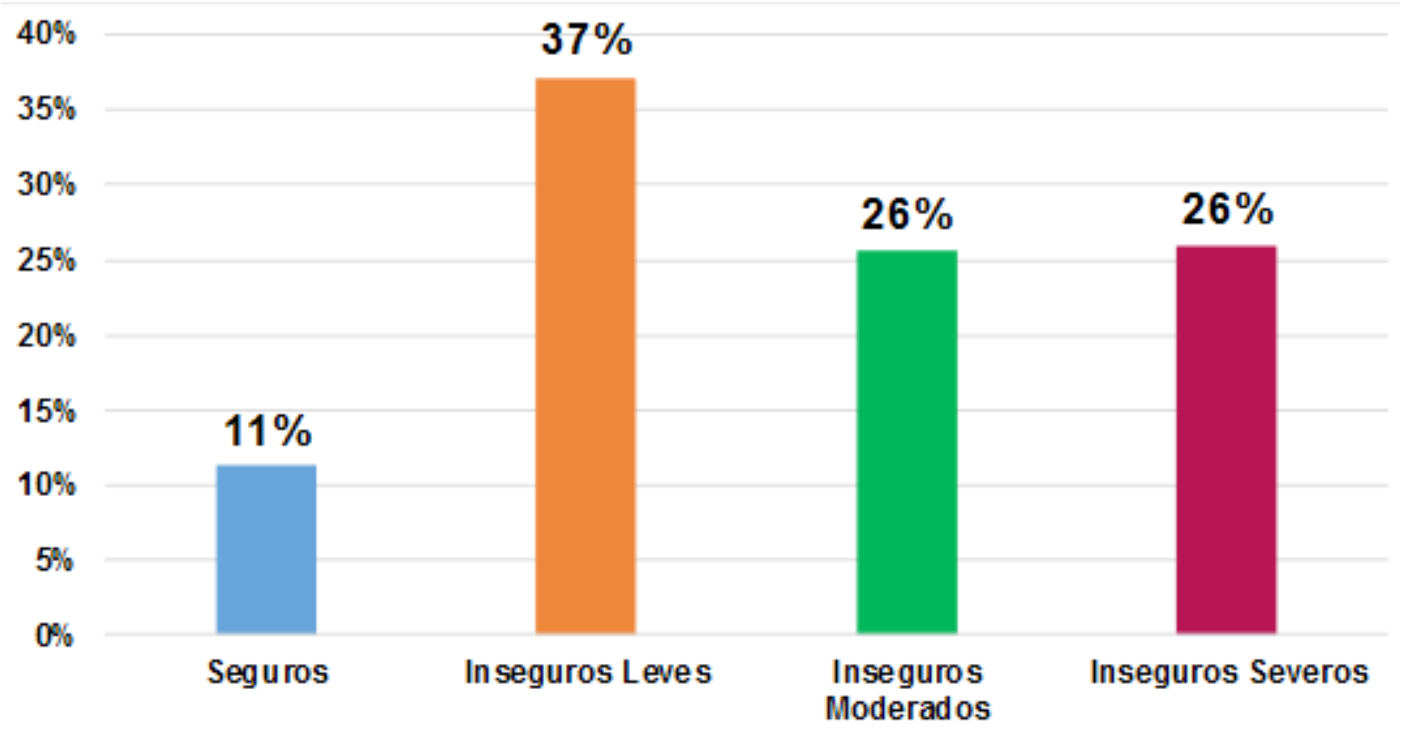

Gráfico 2. Prevalencia de la Inseguridad Alimentaria por categoría de los hogares del municipio San Ramón, 2014-2015. Fuente: Encuesta de Medios de Vida Sostenible y Seguridad Alimentaria y Nutricional 2014-2015

En los hogares visitados, el $11.6 \%$ de ellos solo es integrado por adultos y con menores de 18 años, el $88.4 \%$ de los hogares; con mayores aumentos de inseguridad alimentaria en las categorías leve y moderada en hogares con menores de 18 años y la forma de inseguridad severa en los mayores de 18 años. En general, existe inseguridad alimentaria en los hogares donde hay niños y jóvenes. En ellos se observa la preocupación por los alimentos, la afectación en la calidad y cantidad de alimentos y el padecimiento de hambre. La calidad de la dieta es un determinante común que afectan la salud y el estado nutricional de los miembros de los hogares. La falta de acceso a alimentos básicos y nutritivos por los bajos ingresos de las familias y la limitada producción hogareña de los alimentos son factores que son necesario atender.

Cuadro 1. Tipos de hogares según categorías de Inseguridad Alimentaria en hogares urbanos y rurales del municipio San Ramón Matagalpa, 2014-2015. Fuente: Encuesta de Medios de Vida Sostenible y Seguridad Alimentaria y Nutricional 2014- 2015

\begin{tabular}{|c|c|c|c|c|c|c|c|c|c|c|}
\hline \multirow{3}{*}{$\begin{array}{c}\text { TIPO DE } \\
\text { HOGARES }\end{array}$} & \multicolumn{8}{|c|}{ CLASIFICACIÓN ELCSA } & & \\
\hline & \multicolumn{2}{|c|}{ Seguros } & \multicolumn{2}{|c|}{$\begin{array}{l}\text { Inseguros } \\
\text { Leves }\end{array}$} & \multicolumn{2}{|c|}{$\begin{array}{l}\text { Inseguros } \\
\text { Moderados }\end{array}$} & \multicolumn{2}{|c|}{$\begin{array}{c}\text { Inseguros } \\
\text { Severos }\end{array}$} & \multicolumn{2}{|c|}{ TOTAL } \\
\hline & Casos & $\%$ & Casos & $\%$ & Casos & $\%$ & Casos & $\%$ & Casos & $\%$ \\
\hline $\begin{array}{l}\text { Hogares con } \\
\text { menores de } 18 \\
\text { años }\end{array}$ & 31 & $12 \%$ & 101 & $38 \%$ & 71 & $27 \%$ & 63 & $24 \%$ & 266 & $100 \%$ \\
\hline Hogares adultos & 3 & $9 \%$ & 11 & $31 \%$ & 6 & $17 \%$ & 15 & $43 \%$ & 35 & $100 \%$ \\
\hline Total & 34 & $11 \%$ & 112 & $37 \%$ & 77 & $26 \%$ & 78 & $26 \%$ & 301 & $100 \%$ \\
\hline
\end{tabular}


El patrón de consumo de los hogares visitados, está integrado por 13 alimentos, entre ellos, maíz, sal, arroz, frijol, aceite, café, azúcar, cebolla, huevo, pan dulce, tomate, queso y chiltoma, por más del $50 \%$ de las familias, siendo en su mayorías productos de origen vegetal fuente importante de energía y en menor proporción, de proteínas. El patrón de consumo de la zona geográfica VI integrado por 15 productos en los que incluyen: azúcar, sal, arroz, aceite vegetal, frijol, cebolla, chiltoma y tomate, queso y similares, café en polvo, tortilla de maíz, carne de aves, huevo, bananoplátanos maduros, pan dulce y galletas y papas, todo incluidos en ocho grupos de alimentos de diez que conforma la estructura energética del patrón alimentario nicaragüense. Las formas más comunes de cocinar son leña con el $93 \%$ y gas butano con el $10 \%$.

Cuadro 2. Patrón de consumo de alimentos consumidos por el 50 \% o más de la población del Municipio San Ramón, 2014- 2015. Fuente: Encuesta de Medios de Vida Sostenible y Seguridad Alimentaria y Nutricional 2014- 2015

\begin{tabular}{|l|c|c|}
\hline \multicolumn{1}{|c|}{ ALIMENTOS } & FRECUENCIA & $\%$ \\
\hline Tortilla de maíz & 292 & $97 \%$ \\
\hline Sal, Sal Yodada & 289 & $96 \%$ \\
\hline Arroz & 286 & $95 \%$ \\
\hline Frijol rojo o negro & 277 & $92 \%$ \\
\hline Aceite & 275 & $91 \%$ \\
\hline Café o té & 275 & $91 \%$ \\
\hline Azúcar Blanca & 272 & $90 \%$ \\
\hline Cebolla & 225 & $75 \%$ \\
\hline Huevos de gallina u otras aves & 196 & $65 \%$ \\
\hline Pan dulce (picos, semitas, tortas, bonete, otros) & 193 & $64 \%$ \\
\hline Tomate (frescos o en salsa) & 187 & $62 \%$ \\
\hline $\begin{array}{l}\text { Queso (cualquier tipo, quesillo, cuajada, } \\
\text { requesón, crema otros) }\end{array}$ & 156 & $52 \%$ \\
\hline Chiltoma o chile & 152 & $50 \%$ \\
\hline
\end{tabular}

Entre los medios de vida sostenible, en los hogares sobresale, que el $84 \%$ tiene vivienda propia y $5 \%$ alquilada. El 23\% cuenta con terreno o solar. Entre los bienes del hogar sobresale el uso de teléfono móvil con el $81 \%$, seguido de la radio con $79 \%$ y televisor con el $67 \%$. Un cuarto por ciento de los hogares tiene, además, otros bienes como refrigeradora, licuadora y equipos de sonidos, los dos primeros de importancia para la cocina. El 38\% de los hogares tienen un ingreso menor de C\$2 000 córdobas y el 53\% mayor de C\$2 000 mensual.

El aumento de activos es relevante para la mejora de la seguridad alimentaria de los hogares por lo que contribuye a garantizar las necesidades básicas como la alimentación, nutrición, salud, vivienda, educación entre otros y a su vez, la diversificación de fuentes de ingresos, mayor acceso a los bienes y servicios públicos. 


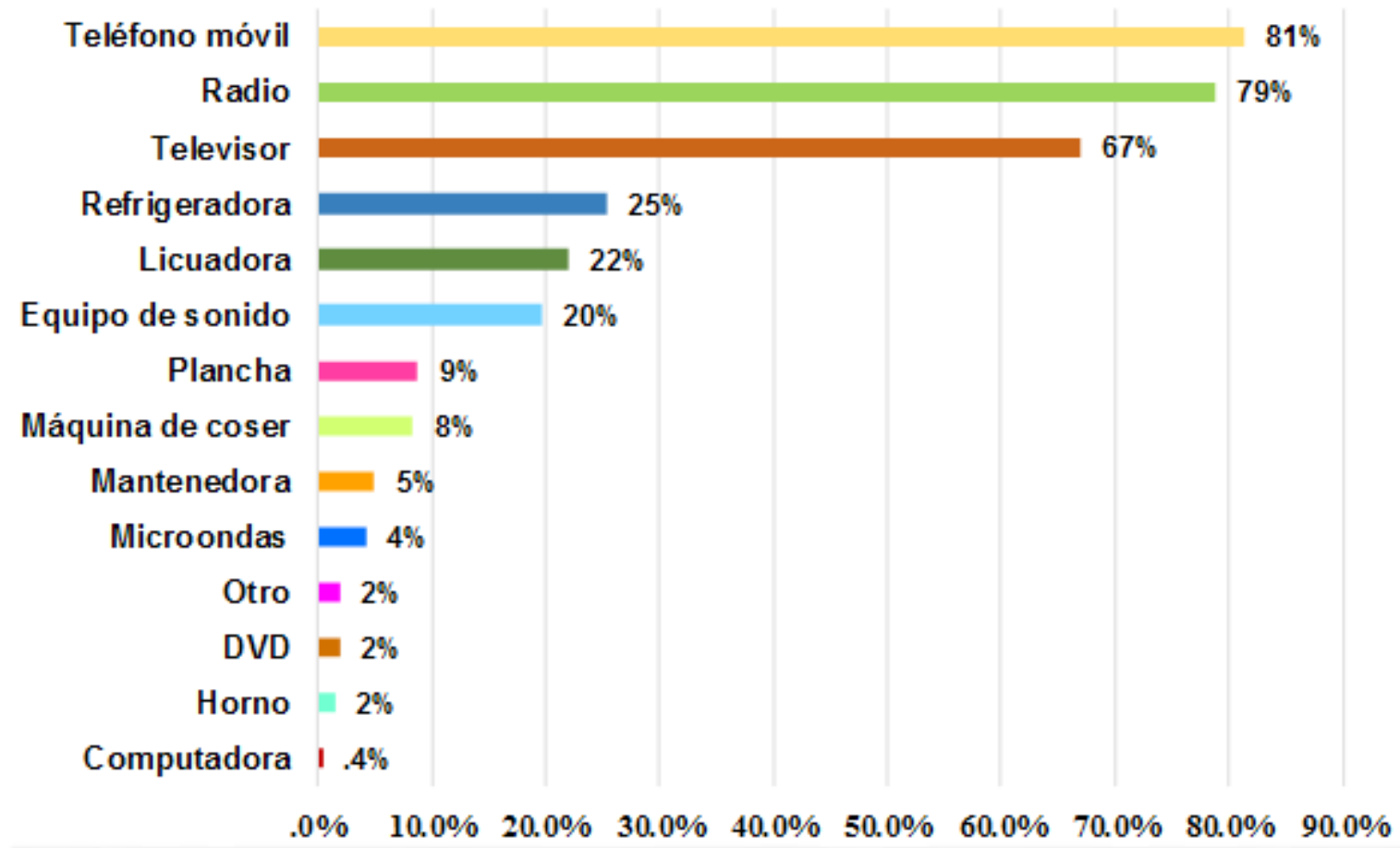

Gráfico 3. Bienes que poseen los hogares de San Ramón 2014-2015 Fuente: Encuesta de Medios de Vida Sostenible y Seguridad Alimentaria y Nutricional 2014- 2015

\section{CONCLUSIONES}

La mayoría de los jefes de hogares son casados con el 41\%, seguido de unión libre con el 32\%, el $50 \%$ son mujeres y el $48 \%$ varones. Siendo la mayoría adultos mayores de 18 años.

La escolaridad con primaria incompleta con el 37\%, seguido de secundaria incompleta con el 15\%, primaria completa con el $11 \%$ y $17 \%$ analfabeta. La ocupación principal es ama de casa con el $29 \%$, seguido de estudiante con el $24 \%$, en tercer lugar, agricultor con el $18 \%$ y jornalero con el $10 \%$, el $5 \%$ empleado no oficinista y $4 \%$ desempleado.

La inseguridad alimentaria representa el $89 \%$ sobresaliendo las categorías moderadas y severas, representando el $52 \%$ y $37 \%$ respectivamente en los hogares. Solo el $19 \%$ tienen seguridad alimentaria.

El patrón de consumo de 23 productos de los hogares, integrado por 13 alimentos, está por debajo de lo normal. Los productos como las carnes y las papas son las menos consumidas.

El 53\% gana un poco más de C\$2 000 al mes para sufragar los gastos de la familia, siendo el 69\% jefe de familia varón y $31 \%$ mujer e integrado por familia extendida.

La falta de acceso a alimentos básicos y nutritivos por los bajos ingresos de las familias y la limitada producción hogareña de los alimentos son factores que son necesario atender.

Entre los medios de vida sostenible en los hogares sobresalen, $84 \%$ tienen vivienda propia con el $84 \%$ y $5 \%$ alquilada. Cuenta el $23 \%$ de ellos con terreno o solar. 


\section{REFERENCIAS BIBLIOGRÁFICAS}

Alcaldía de San Ramón. (2005). Caracterizacion del muicipio San Ramón. Matagalpa.

Baumeister, E. (1999). Caracterĺsticas y potencial de la agricultura Nicaragüense.

CEPAL. (2007). Desigualdades sociodemogràficas en Nicaragua: tendencias, relevancia y politicas pertinentes.

FAO. (2002). Concepto de seguridad alimentaria.

FAO. (2007). Guía de Seguridad Alimentaria y Nutricional para el uso del personal agropecuario de Nicaragua. Managua: FAO.

FAO/OHIO State University. (2012). Informe sobre la aplicación pasada y presente de la Escala latinoamericana y caribeña de Seguridad Alimentaria y otras herramientas similares en América del Norte, Centroamérica y el Caribe. Disponible en: http://www.insp.mx/elcsa/ docs

GRUN. (2011). Plan Nacional de Desarrollo Humano 2012-2016. Managua.

FPRI. (2013) Indice Global del Hambre. El reto del hambre: construir resilencia para mejorar la SAN. 66p.completo. Washington, Estados Unidos. Disponible en: http://www.infpri.org/sites/difault/ files/publications/ghi13pdf
IMFEWS-Acción contra el Hambre. (2010). Perfiles de Medios de Vida. Sistema Mesoamericano de Alerta Temprano para la Seguridad Alimentaria (ACF). Nicaragua. Disponible en: http:// es.slideshare.net/

MAGFOR-INCAP. (2004). Informe Final de IV Encuesta Nacional de Consumo de Alimentos. Managua.

MARENA. (2008). Plan de ordenamiento y manejo de la subcuenca prioritaria: Análisis, Síntesis y Propuesta de uso Agroforestal. Managua.

Scheneider, S. (2006). Territorio y enfoques territoriales. Buenos Aires.

Siles, G. (2014). Uso y manejo del agua en el sistema ecoforestal, Comarca de Pochocuape. Managua.

PRESANCA. (2010). Análisis de situación de Seguridad Alimentaria y Nutricional en Centroamérica y República Dominicana. Informe técnico 208pp. Programa Regional de SAN 